\title{
Lifestyle-Medizin und Wunschbehandlungen
}

\author{
Von modernen Wunderpillen und Operationssucht: \\ Auf der Suche nach dem ewigen Jungbrunnen und Arcanum
}

W. Harth

\author{
Lifestyle Medicine and Treatment on Demand - Modern Wonder Pills and \\ Polysurgical Addiction in Search of the Arcanum and the Fountain of Youth
}

\section{Zusammenfassung}

Die ärztliche Tätigkeit hat in den letzten Jahren eine deutliche Erweiterung der Möglichkeiten von Wunschbehandlungen im Bereich der Medizin erfahren. Besonders in der Dermatologie hat eine zunehmende Nachfrage und Inanspruchnahme von ärztlichen/medizinischen Dienstleistungen für Gesunde $\mathrm{zu}$ einem drastischen Wandel hin zur kosmetischen Dermatologie geführt. Einer immer breiteren Bevölkerungsschicht ist es aufgrund der verbesserten ökonomischen Situation möglich geworden diese medizinisch-ästhetischen Verfahren in Anspruch zu nehmen. Schönheitsoperationen und Lifestyle-Medikamente stehen dabei im Vordergrund von Angebot und Nachfrage. Begleitet wird der steigende Bedarf durch Werbemaßnahmen und immer wiederkehrende Berichte in den privaten Printmedien, Fernsehsendern und Internet. Dabei wechseln modebedingt ständig die Schönheitsideale und erzeugen somit eine neue Nachfrage. Neben dem ärztlichen Angebot besteht auch ein breites Angebot von nicht-ärztlich tätigen Leistungsanbietern und eine breite Subkultur von paramedizinischen Laienanwendungen. Der Bezug aller Lifestyle-Medikamente ist heute praktisch uneingeschränkt über das Internet möglich und ein Medikamentenmissbrauch ohne ärztliche Indikation kaum beherrschbar. Durch eine zunehmende Globalisierung sind weiterhin Operationen im Ausland keine Grenzen gesetzt.

Mögliche Risiken sowie Komplikationen der Verfahren werden dabei von den Konsumenten oftmals verdrängt oder Nebenwirkungen und Wechselwirkungen geleugnet. Subjektive Körperbildstörungen, Minderwertigkeitsgefühle und Soziophobien
Abstract

Medical activity in recent years has experienced a marked expansion of possibilities for lifestyle medicine and treatment on demand. Especially in dermatology, an increasing demand for and use of doctor/medical services for healthy persons has led to a drastic change up to cosmetic dermatology. An ever broader segment of the population is in a position to make use of these medical-aesthetic procedures thanks to their improved economic situation. Cosmetic surgery and lifestyle medications are in the forefront of supply and demand. This increasing demand is awakened by advertising and repeated reports in private press, television shows and especially in the internet with constantly changing, fashion-dictated ideals of beauty. In addition, there is a broad subculture of paramedical lay applications. Today it is possible to buy all lifestyle medications, practically without limit, through the internet, and drug abuse without medical indication is almost impossible to control. Due to increasing globalisation, there is no limit to operations in foreign countries.

Possible risks and complications of the procedures are pushed aside and side effects and interactions denied. Subjective impairments in appearance, feelings of inferiority and sociophobias may be at the forefront of somatising complaint symptoms. The emotional disorders behind them, such as body dysmorphic disorder or polysurgical addiction often remain undiscovered and should find increased attention in the entire area of treatment on demand.

Klinik für Dermatologie und Phlebologie, Vivantes Klinikum Berlin, Friedrichshain; „Akademisches Lehrkrankenhaus der Charité - Universitätsmedizin Berlin“ (Chefärztin: PD. Dr. med. B. Hermes)

Korrespondenzadresse

PD. Dr. med. Wolfgang Harth · Stellvertretender Klinikdirektor · Leitender Oberarzt · Klinik für Dermatologie und Phlebologie · Vivantes Klinikum Berlin, Friedrichshain · Landsberger Allee $49 \cdot 10249$ Berlin .

E-mail: wolfgang.harth@vivantes.de 
können im Vordergrund einer somatisierten Beschwerdesymptomatik stehen. Dahinterstehende psychische Störungen wie eine körperdysmorphe Störung oder eine Operationssucht bleiben oft unentdeckt, und sollten im gesamten Bereich der Wunschbehandlungen mehr Beachtung finden.

\section{Einleitung}

Der Gesundheitsstatus der Bevölkerung hat sich in den letzten Jahrzehnten stetig verbessert. Gleichzeitig ist in den westlichen Industrienationen die Anspruchsforderung an die Medizin gestiegen, und gewinnt dabei ein Schönheitsbedürfnis in unserer Gesellschaft zunehmend an Bedeutung. Freizeit, Lebensgenuss, Spaß und Selbstverwirklichung mit Attraktivität, Jugendlichkeit und Erfolg werden besonders von der jüngeren Generation als Lebensziele definiert. Wer jedoch Normvarianten aufzeigt oder in die Jahre kommt, hat Mühe den jugendlichen Lifestyle aufrecht $\mathrm{zu}$ erhalten und versucht Abweichungen entgegenzuwirken [1]. Die aktuellen Idealvorstellungen in den westlichen Industrienationen führen zu einer immer breiteren Subspezialisierung im Bereich der medizinischen Kosmetologie. Dabei werden medizinische Verfahren und Forschungswissen sowie spezielle Forschungsanstrengungen zur Entwicklung neuer Laser- oder Lifestyle-Medikamente einschließlich invasiver operativer Methoden im Dienst der Verjüngung und Schönheit eingesetzt.

Insgesamt rund 180000 ästhetisch kosmetische Eingriffe haben allein nur die Mitglieder der Gesellschaft für ästhetische Chirurgie Deutschland e.V. im Jahr 2003 durchgeführt [2]. Im Vordergrund stehen dabei Faltenbehandlung per Laserchirurgie und Botolinumtoxin, Liposuktion, Ausfüllen von Falten sowie Venenchirurgie, Lidkorrektur, Brustvergrößerung, Nasenkorrektur, Ohrkorrektur, Brustverkleinerung, Haarverpflanzung und Facelift. Oftmals hat der betroffene Personenkreis genaue Vorstellungen über die gewünschten Verfahren und erfolgt der Arzt-Patientenkontakt mit klarer Absicht einer Wunschtherapie. Dabei wird nur in den seltensten Fällen nach Nebenwirkungen der angewendeten Methoden gefragt, welche billigend wie in keinem anderen Bereich der Medizin in Kauf genommen werden. Hierzu gehören Komplikationen beispielsweise nach Liposuktion. $\mathrm{Zu}$ den typischen Risiken zählen dabei Asymmetrie, Hautdellen, Hautverfärbungen, Sensibilitätsstörungen, Infektionen, Serome, Narbenbildung, Blutungen. Schwerwiegende Komplikationen wie Lungenembolie, hämatogener Schock, Hautnekrosen, Verletzung innerer Organe, nekrotisierende Faszitis, Sepsis treten in $0,1 \%$, Todesfälle in $0,2 \%$ der Fälle auf [3].

\section{Anpassungsstörungen}

Bei objektivierbaren entstellenden Krankheiten (Autoimmundermatosen, Atopisches Ekzem, Acne conglobata), posttraumatischen hypertrophen Narben, Keloiden oder entstellenden Neoplasien liegen häufig Anpassungsstörungen vor. Es besteht ein subjektives Leiden, emotionale Beeinträchtigung und Einschränkung der Lebensqualität. Das psychische Bild der Anpassungsstörung ist heterogen und meist durch depressive Reaktionen sowie Angst auch in Form einer gemischten Symptomatik gekenn- zeichnet. Als zentraler Mechanismus zeigt sich immer wieder, dass Entstellungen zu einer Anpassungsstörung mit Angst - Depression und letztendlich sozialem Rückzug oder Soziophobie führen kann. Eine ästhetische Operation der Entstellung kann dann durchaus auch zur Heilung der psychischen Störung führen [4].

\section{Körperdysmorphe Störung}

Andererseits werden in der kosmetischen Medizin nicht nur Patienten mit klar definierten Symptomen vorstellig, sondern es kann ein so genanntes Hässlichkeitssyndrom im Sinne einer körperdysmorphen Störung $[5,6]$ vorliegen, wobei trotz fehlenden objektivierbaren Befunden dennoch ein subjektives Entstellungsgefühl besteht.

Körperdysmorphe Störungen bezeichnen als zentrales Kriterium die übermäßige Beschäftigung mit einem Mangel oder einer Entstellung im körperlichen Aussehen. Dieser Mangel ist entweder überhaupt nicht vorhanden oder nur äußerst gering ausgeprägt [7,8]. Hinzu kommen eine ständige Kontrolle des Aussehens vor dem Spiegel und aufwändige Pflegemaßnahmen.

Die Prävalenz der körperdysmorphen Störung wird mit ca. 1\% der amerikanischen Gesamtbevölkerung und bis $4 \%$ bei amerikanischen und deutschen Studenten [9] angegeben sowie bis $15 \%$ bei kosmetisch-chirurgischen Wunschbehandlungen [10].

Verschiedene Theorien versuchen die Entstehung einer körperdysmorphen Störung verstehbar zu machen. Psychodynamische Erklärungsmodelle gehen besonders von Störungen im „SelbstIdeal“ und im „Selbst-Sein-Wollen“, unsicheren Bindungsmustern, Minderwertigkeitsgefühlen, selbstentwertendem Ekelgefühl oder Selbstwertzweifel aus [11]. Zusätzlich können medieninduzierte Faktoren durch die Engrammierung von dargestellten Schönheitsidealen prädisponieren [12]. Patienten mit körperdysmorpher Störung suchen in verstärktem Maße nach somatischen Wunschbehandlungen. Durch die Operation soll dann vermeintlich ein psychisches Gleichgewicht hergestellt oder stabilisiert werden. Die Fehleinstellung beinhaltet, dass unbewusst versucht wird auf Organebene eine Scheinlösung zu erreichen, die durch den operativen Eingriff allein nicht gelingen kann. Häufig wird eine dahinterliegende psychische Störung von den Betroffenen jedoch nicht eingesehen und kann nicht akzeptiert werden [13]. Da die Ästhetik in besonderem Maße einer subjektiven Beurteilung unterliegt, kann bei Vorliegen einer körperdysmorphen Störung postoperativ ein anderes Aussehen von den Patienten als ungewohnt und störend empfunden werden. Psychisch gestörte Patienten neigen dann zu abwertenden Haltungen. Aus der praktischen Erfahrung hat sich gezeigt, dass solche Problempatienten - selbst bei gutem Ergebnis (Abb.1) - oft ungerechtfertigt aktiv eine schlechte Mundpropaganda betreiben, die dem Chirurgen langfristig schadet [14]. Schon allein aus diesem Grunde sollte die OP-Indikation in der ästhetischen Medizin hinsichtlich dem Vorliegen einer psychischen Störung einschließlich Suizidalität im Vorfeld genau geprüft werden. Invasive Eingriffe einer anderen Person zuliebe sollten strikt abgelehnt werden [15]. Vor Gefälligkeitsoperationen muss gewarnt werden. 


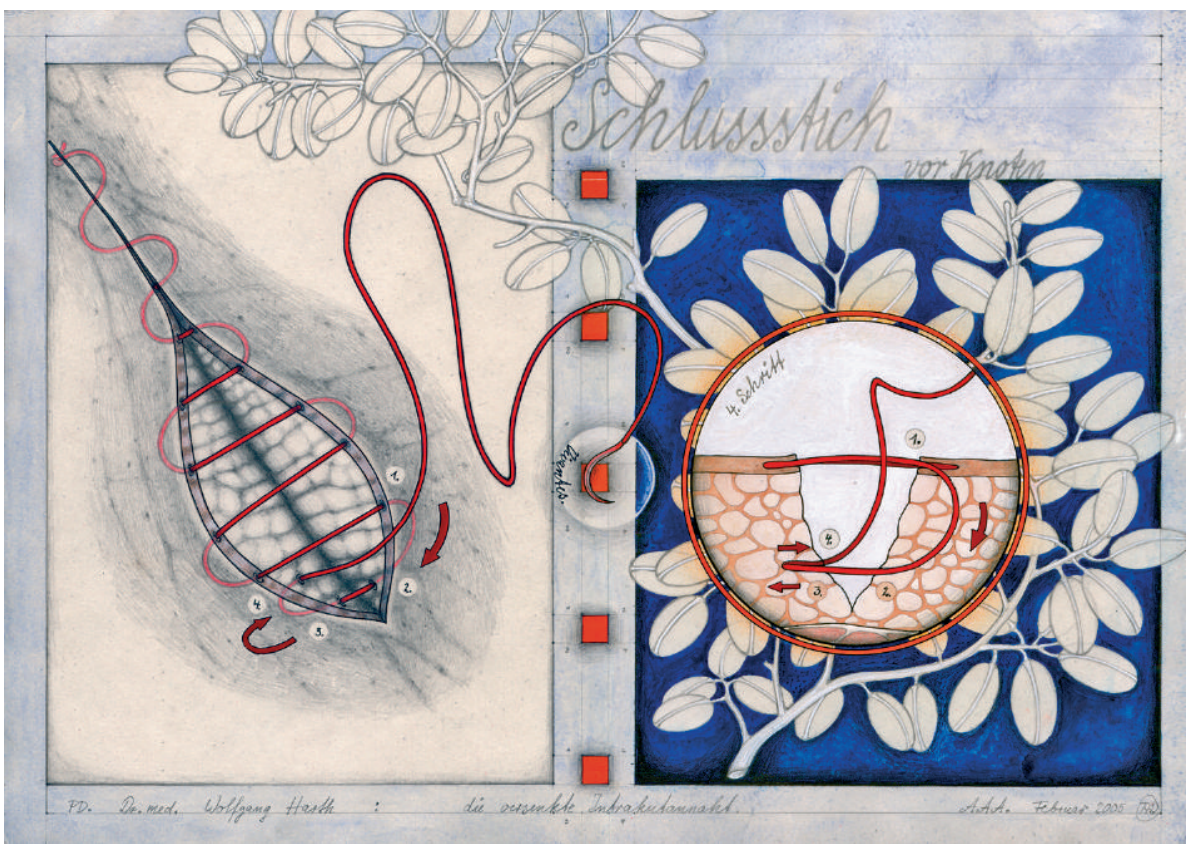

Abb. 1 Ästhetische Operation mittels versenkbarer Intrakutannaht (Graphik T. Nicolai Erfurt).

Chirurgen sollten bei dramatisierenden und zugleich vagen Symptombeschreibungen hellhörig werden. Oftmals ist das Gefühl, dass „etwas nicht stimmt“ oder auch die Wut in der Gegenübertragung als Alarmsignal wegweisend (Tab. 3), um die Indikation erneut zu überprüfen, im Expertenteam zu diskutieren oder eine weiterführende Betreuung beispielsweise in einer Liaisonsprechstunde einzuleiten [16]. Generell muss bei ästhetischen Eingriffen im Vergleich zu vital indizierten Operationen eine intensivere OP-Aufklärung und strengste Indikationsstellung aufgrund möglicher Komplikationen durchgeführt werden, auch um übertriebene Erwartungen ähnlich wie in der Reproduktionsmedizin zu korrigieren.

\section{Operationssucht (polysurgical addiction)}

Im Gegensatz zur Angst vieler Patienten vor invasiven Eingriffen scheinen einige Patienten generell Operationen zu begrüßen. Musste man früher oft mit der Operationsscheu kämpfen, so kämpft man heute mit der Operationsneigung bzw. Operationswut (ehemaliges Synonym:,Mania operativa“) [17], besonders auch bei kosmetischen Wunschoperationen. Die Diagnose eines nicht indizierten Operationswunsches kann schon aus der Anamnese mit multiplen unklar begründeten Voroperationen nahe liegen. Das Drängen auf einen chirurgischen Eingriff kann unbewusst auf der Grundlage einer körperdysmorphen Störung oder Zwangsstörung entstehen oder den Wunsch nach Selbstverstümmelung, Selbstbestrafung bzw. partialen Suizid - ähnlich wie bei Patienten mit artifiziellen Störungen und MünchhausenSyndrom - ausdrücken. Tiefenpsychologisch wurden Körperbildstörungen, Minderwertigkeitsgefühle mit Selbstwertproblematik, Schamgefühle und Ekel sowie sadomasochistische und suizidale Tendenzen beschrieben [18]. Oft treten Spaltungsphänomene auf, wobei die Vorstellung eines guten und bösen Bezirks im eigenen Körper existiert. Das Böse soll dann vom Chirurgen entfernt werden, so dass nur das Gute verbleibt. Alle psychischen und physischen Körperschäden werden dabei dem ne- gativen Körperteil zugeschrieben. Solche dissoziativen Phänomene treten besonders dann auf, wenn frühe Gewalterfahrungen vorliegen, die eine Spaltung zur maximalen Abwehr erfordert [19].

Weiterhin können Patienten den dramatischen Event einer Operation genießen, aufgrund der ihnen zuteil werdenden Aufmerksamkeit durch das Behandlungsteam oder von Familie und Umfeld. Der Chirurg wird dann Werkzeug eines psychopathologischen Lösungsversuches und geht nach anfänglicher Idealisierung die Gefahr einer enormen Wut des Patienten ein, wenn er sich der Wunschbehandlung verweigern sollte. Unabhängig davon, ob die Operation erfolgt oder durch den Chirurgen abgelehnt wird, kann es im weiteren Verlauf zu einer aktiven Inszenierung mit so genanntem „Koryphäen-Killer“-Verhalten kommen, damit sich der Patient aus der Rolle des passiv Leidenden befreien kann [20].

\section{Lifestyle-Medikamente}

Scheinbar harmloser werden konservative, meist medikamentöse Wunschbehandlungen bewertet. Lifestyle-Medikamente werden in diesem Zusammenhang auch verniedlichend als smart pills bezeichnet.

Im Bereich der gesamten Medizin haben Lifestyle-Medikamente in den letzten Jahren eine zunehmende Bedeutung erlangt [21]. Dabei handelt es sich um Substanzen, die zur Erhöhung des Wohlbefindens und des individuellen Lebensgenusses bzw. der Lebensqualität eingenommen werden. Die Einnahme wird weiterhin durch Modetrends sowie aufgrund von Eigenheiten der privaten Lebensführung (Lifestyle) beeinflusst.

Definition: Lifestyle-Medikamente sollen eine trendabhängige, individuelle Lebensqualität verbessern, ohne dass eine Krankheit oder entsprechende Therapieindikation vorliegt. 
Tab. 1 Lifestyle-Medikamente (nach Hesselink 1999)

\begin{tabular}{|c|c|c|c|c|}
\hline Nootropica & Psychopharmaka & Hormone & „Ecodrugs“ & sonstige Pharmaka \\
\hline Dimethylaminoethanol/DMAE & Gammahydroxybutyrat (GHB) & Dehydroepiandrosteron (DHEA) & Absinth & Dextromethorfan (DXM) \\
\hline Hydergin & Ketamin & Pregnenolon & Echinacea & Metformin \\
\hline Piracetam & Fluoxetin & Melatonin & Kava-Kava & Propranolol \\
\hline Pramiracetam & Selegilin & Desmopressin & Herbal exstacy & Koenzym Q \\
\hline Acetyl-I-Carnitin & S-Adenosyl-Metionin (SAM) & Norethisterone (Zyklusregulation) & Ritual spirit & Protonenpumpen-Inhibitoren \\
\hline Oxiracetam & Methylphenidat & Kontrazeption & Guarana & Nimodipin \\
\hline Aniracetam & Adrafinil/Modafinil & Wachstumshormon & Organpräparate & Centrophenoxin \\
\hline Vinpocetin & Sibutramin & Anabolika & Johanniskraut & Clenbuterol \\
\hline Idebenon & I-Tryptophan & & Vitamine & $\mathrm{NADH}$ \\
\hline Vincamin & Serotonin & & Mineralien & Phenytoin \\
\hline Cyprodenat & Dexfenfluramin & & Aminosäuren & Deprenyl \\
\hline \multirow[t]{3}{*}{ Yohimbin } & Ecstacy (XTC) & & Gingko biloba & Bupropion \\
\hline & Ondansetron & & & Orlistat \\
\hline & Parlodel & & & Crestor, Simvastin \\
\hline
\end{tabular}

Dabei werden teilweise harmlose oder vorübergehende Symptome (Blähungen, Erektionsstörungen) sowie physiologische Körperfunktionen (Schwitzen, Haarzyklus, Herzschlag) von einigen Gesunden als Krankheiten angesehen. Teilweise besteht die unberechtigte Befürchtung von Mangelerscheinungen (Vitamine) oder werden physiologische Alterungsprozesse geleugnet. Dahinter können hypochondrische Störungen und im Speziellen körperdysmorphe Störungen bei einer vorwiegenden Beschäftigung mit der äußeren Erscheinung stehen. Differenzialdiagnostisch müssen Somatisierungsstörungen mit rezidivierenden, multiplen, klinischen Beschwerden (gastrointestinale, sexuelle und pseudoneurologische Symptome) sowie Zwangsstörungen abgegrenzt werden. Weiterhin können psychosomatische Probleme als rein somatische Krankheiten angesehen werden. Bei solchen somatoformen Störungen erfolgt die wiederholte Darbietung körperlicher Symptome in Verbindung mit hartnäckigen Forderungen nach medizinischen Untersuchungen trotz wiederholt negativer Ergebnisse und Versicherung der Ärzte, dass die Symptome nicht körperlich begründbar sind. Eine Medikalisierung des physiologischen Lebens soll dann - jedoch erfolglos psychosoziale Probleme lösen. Dabei werden bekannte und häufige Nebenwirkungen von den Anwendern bewusst in Kauf genommen. So wünschen beispielsweise im Rahmen ästhetischer Verfahren auch ökologisch bewusst lebende Personen ohne größere Bedenken eine Anwendung von Botulinumtoxin, eines der weltweit stärksten Nervengifte.

Besonders abzulehnen ist die Lifestyle-Anwendung von verschreibungspflichtigen Medikamenten („Medikamente für Gesunde“), deren Einnahme medizinisch nicht indiziert ist. Es handelt sich um einen Medikamentenmissbrauch im erweiterten Sinne. Da hierfür eine ärztliche Konsultation notwendig ist, werden die Ärzte zwecks Rezeptierung der Wunschmedikation aufgesucht. Das Einfordern der Therapie von Gesunden, besonders aber wenn eine psychische Störung nicht sicher auszuschließen ist, setzt den Arzt unter emotionalen Druck in der Ambivalenz zwischen Drängen und nicht gegebener Indikation. Zentral und wichtig ist die adäquate Indikationsstellung im Vorfeld, wobei der Arzt im Zweifelsfalle die Wunschbehandlung ablehnen sollte.
Tab. 2 Häufige Lifestyle-Medikamente

\begin{tabular}{|c|c|c|}
\hline Arzneimittel & Indikation & Lifestyle-Missbrauch \\
\hline $\begin{array}{l}\text { Botulinum-Toxin, } \\
\text { Methanthelinium- } \\
\text { bromid }\end{array}$ & Hyperhidrosis & $\begin{array}{l}\text { Abstellen des normalen } \\
\text { belastungsabhängigen Schwit- } \\
\text { zens, körperdysmorphe Störung, } \\
\text { Soziophobie, Schamstörung }\end{array}$ \\
\hline $\begin{array}{l}\text { Isotretinoin/ } \\
\text { Tretinoin }\end{array}$ & Acne vulgaris & $\begin{array}{l}\text { Dorian-Gray-Syndrom } \\
\text { (Traum nach ewiger Jugend), } \\
\text { Stopp einer normalen Seborrhö }\end{array}$ \\
\hline $\begin{array}{l}\text { Metformin, Crestor, } \\
\text { Simvastatin, Orlistat, } \\
\text { Sibutramin }\end{array}$ & $\begin{array}{l}\text { Adipositas, } \\
\text { Diabetes, Hyper- } \\
\text { cholesterinämie }\end{array}$ & $\begin{array}{l}\text { Anorexia nervosa, } \\
\text { Sisi-Syndrom }\end{array}$ \\
\hline $\begin{array}{l}\text { Minoxidil, } \\
\text { Finasterid }\end{array}$ & $\begin{array}{l}\text { androgenetische } \\
\text { Alopezie }\end{array}$ & $\begin{array}{l}\text { körperdysmorphe Störung } \\
\text { bei regelrechtem Befund }\end{array}$ \\
\hline Modafinil & Narkolepsie & Müdigkeit \\
\hline Paroxetin, Fluoxetin & depressive Störung & Schüchternheit, Scham \\
\hline $\begin{array}{l}\text { Sildenafil, Tadalafil, } \\
\text { Phentolamine, } \\
\text { Apomorphine }\end{array}$ & erektile Dysfunktion & $\begin{array}{l}\text { ewige Potenz und } 100 \% \\
\text { kontrollierbare Erektion }\end{array}$ \\
\hline Somatotropin & $\begin{array}{l}\text { hypophysärer } \\
\text { Kleinwuchs }\end{array}$ & Jungbrunnen, Doping \\
\hline Testosteron & Testosteronmangel & Midlife Crisis \\
\hline
\end{tabular}

Tab. 3 Alarmsignale in der psychosomatischen Chirurgie

Übertreibung des körperlichen Defektes

Störungen des Selbstbildes

körperdysmorphe Störung

unklare Motivation oder zu hohe Erwartungen an die Therapie

affektive Störung und Angststörungen

Somatisierungsstörungen (multiple weitere Beschwerden)

akute Psychose

schwerwiegende narzisstische Persönlichkeitsstörungen/Borderline-Störung

Therapie einer anderen Person zuliebe 
Missbräuchlich von Gesunden verwendete Medikamente werden aber auch teilweise ohne ärztliche Indikationsstellung als Lifestyle-Medikamente eingenommen, unter Simulation von Beschwerden oder als Gefälligkeitsrezept verschrieben. Zahlreiche Vertriebssysteme bieten über Internet oder Spezialgeschäfte unterschiedliche Substanzen teilweise illegal an. Inzwischen kann fast jeder jedes Medikament außerhalb der ärztlichen Kontrolle über den Schwarzmarkt und dessen weltweite Vertriebssysteme beziehen.

Zu den Hauptgruppen von Lifestyle-Medikamenten zählen Nootropica, Psychopharmaka, Hormone und Ecodrugs, die zur Behandlung der erektilen Dysfunktion, Steigerung der sexuellen Potenz, der Raucherentwöhnung, der Abmagerung beziehungsweise Zügelung des Appetits, der Regulierung des Körpergewichts sowie der Verbesserung des Haarwuchses dienen [22] (Tab.1). In der Dermatologie steht die Einnahme von LifestyleMedikamenten zur Beeinflussung kosmetischer Befunde, die meist lediglich Folge natürlicher Alterungsprozesse der Haut sind, im Vordergrund (Tab. 2).

Übergewicht ist ein zentrales Problem unserer Wohlstandsgesellschaft und kann seit einigen Jahren mit Orlistat und Sibutramin behandelt werden. $\mathrm{Zu}$ dem beliebten Lifestyle-Missbrauch in diesem Bereich gehören neuerdings auch Substanzen wie Antidiabetika (Metformin) und besonders Lipidsenker zur Gewichtsreduktion. Eine breite Masse hat die medikamentösen Cholesterinbremsen (Simvastatin) entdeckt, um auch ohne ausgewogene Ernährung, nach fettreichen Exzessen und unkontrollierten Essorgien („Die Pille für das Fett danach“) den Fettspiegel zu senken. Die Gefahr ist das Gegenteil der langjährigen Anstrengungen im Bereich präventiver Maßnahmen. Aufgrund der scheinbaren Sicherheit einer Medikamenteneinnahme werden entgleiste Stoffwechsel und unnötige Medikamentennebenwirkungen generiert.

Strikt abzulehnen sind weiterhin Hormonkuren mit Wachstumshormonen als vermeintlicher Jungbrunnen [23]. Wachstumshormone wie Somatropin werden heute gentechnisch hergestellt und stehen in ausreichender Menge günstig zur Verfügung. Als Lifestyle-Droge soll Somatropin verjüngen und schlank machen, jedoch ist die Wirkung einer Substitution im Alter nicht bewiesen. Trotzdem finden Wachstumshormone aktuell eine breite Anwendung zum Muskelaufbau, Körperfettabbau, Abnahme der Faltenbildung, Energiezunahme und Besserung der vita sexualis. Folgewirkungen wie Diabetes mellitus oder eine möglicheTumorinduktion sind aktuell noch nicht abzuschätzen.

Psychopharmaka wie Fluoxetin werden ohne Indikation zur Verbesserung der Geselligkeit, Gewichtsreduktion, Enthemmung oder Antriebssteigerung als Lifestyle-Medikamente eingenommen. Neu ist der großzügige Einsatz von Paroxetin als „Pille für die Stillen“ bei Soziophobie. Abzulehnen ist in diesem Zusammenhang die missbräuchliche Lifestyle-Einnahme von Methylphenidat $\left(\right.$ Ritalin $^{\circledR}$ ) oder Atomoxetin, welches beim Aufmerksamkeitsdefizit Syndrom Verwendung findet. Zur Verlängerung der Wachperioden wird missbräuchlich Modafinil, ein Medikament zur Behandlung der Narkolepsie als Lifestyle-Droge eingenommen, welches aber in Deutschland BTM-pflichtig ist. Inzwischen sollen 3-10\% der Studenten stimulierende Medikamente wie Methylphenidat missbräuchlich einnehmen, um während der Prüfungsvorbereitung abends länger wach bleiben zu können [24].

Kaum ein anderes Medikament hat wie der Phosphodiesteraseinhibitor Sildenafil zur Therapie der erektilen Dysfunktion bei seiner Markteinführung 1998 eine weltweit so breite und öffentliche Diskussion des privaten Sexualverhaltens ausgelöst. So konnte in diesem Zusammenhang durch die Enttabuisierung jedoch auch eine Somatisierung von psychosozialen Ursachen der erektilen Dysfunktion beobachtet werden. Aus einem bislang „normalen“ gelegentlichen Versagen wurde eine „Volkskrankheit“" [25], da die männliche Bevölkerung immer schon nach dem irrealen Wunschdenken einer 100\% steuerbaren Erektion strebt. Nebenwirkungen oder Wechselwirkungen mit Todesfolge sowie Erblindung müssen bei der ärztlichen Indikationsstellung berücksichtigt und können bei privatem Missbrauch kaum abgeschätzt werden [26].

Inzwischen stehen weitere Lifestyle-Medikamente wie Tadalafil, Vardenafil teilweise mit längerer Wirkungsdauer im Sinne einer „Wochenendpille“ sowie Apomorphine zur Verfügung. Auf der Suche nach weiteren Substanzen zur Steigerung der Libido wird derzeit die hormonähnliche Substanz PT-141 entwickelt, die ähnlich wie die Apomorphine auf das Gehirn wirkt.

Neu ist die Thematik der „Alternde Mann“, wobei einer Testosteron-Abnahme außer Libidoverlust zusätzlich eine unbewiesene Vielzahl von Allgemeinbeschwerden zugeschrieben werden. In diesem Zusammenhang werden Testosteron-Depot-Präparate neuerdings mit nur viermaliger i.m.-Applikation (Nebido $\left.{ }^{\circledR}\right)$ im Jahr zur Substitution von nachgewiesenen besonders genetisch bedingten Mangelsyndromen eingesetzt. Seit 2003 die Gel-Applikationsform zur Verfügung steht, erfolgt oftmals eine missbräuchliche Anwendung auch ohne nachweisbaren pathologisch erniedrigten Testosteronspiegel als Lifestyle-Medikament. Der Einsatz gegen eine Midlife Crisis ist abzulehnen.

Fälschlicherweise auch als „Viagra für die Frau“ bezeichnet, wird aktuell die Zulassung von Testosteron-Pflastern als Intrinsa ${ }^{\circledR}$ zur Luststeigerung für Frauen diskutiert.

Haarausfall und besonders die weit verbreitete androgenetische Alopezie bei Männern ist ein häufiger Grund für die Konsultation eines Dermatologen. Seit der Einführung von Finasterid werden in diesem Zusammenhang aber auch vermehrt Patientenvorstellungen mit körperdysmorphen Störungen bei vollem Haarkleid beobachtet. In diesem Bereich ist zukünftig mit zahlreichen neuen Markteinführungen zu rechnen (Dutasterid: 5-alpha Reduktase Typ I und II, Latanoprost).

Botulinumtoxin ist ein Neurotoxin und findet breite Anwendung in der kosmetischen Medizin bei Falten- und Schweißbildung. Beim Off-label-Einsatz von Botulinumtoxin handelt es sich fast immer um eine Lifestyle-Medikation par excellance. Bei einer körperdysmorphen Störung mit normwertig physiologischem Schwitzen wurde kürzlich als neue Diagnose die Bezeichnung „Botulinophilie“ etabliert [27]. 
Bedenklich ist der Einsatz von Niedrigdosis-Isotretinoin, um eine physiologische Seborrhö abzustellen und so eine gelegentlich glänzende Stirn zu vermeiden. Die Nebenwirkungen, besonders Teratogenität und Stoffwechselstörungen, stehen dabei in keinem Verhältnis zur erwünschten Wirkung als Lifestyle-Medikament.

Zusammenfassend können Wunschoperationen oder Wunschbehandlungen mit Lifestyle-Medikamenten als Ersatzlösung für psychische Probleme wie beim Dorian-Gray-Syndrom [28] oder der Botulinophilie erachtet werden, wobei die Betroffenen zugrunde liegende psychosoziale Konflikte verdrängen. Bei der körperdysmorphen Störung ist der Einsatz von Lifestyle-Medikamenten kontraindiziert. Meist stehen depressive Störungen, Angststörungen sowie Zwangsstörungen im Vordergrund. Oft besteht eine Soziophobie. Als Auslöser können eine narzisstische Kränkung und Schamgefühle im Umgang mit anderen oder in der Öffentlichkeit von Bedeutung sein. Hohe Leugnung und großer Widerstand gegenüber psychosomatischen Erklärungsmodellen gehen fast immer mit einer rein somatischen Behandlungserwartung oder auch Einforderung einer Wunschbehandlung einher. Teilweise gelingt dann bei somatoformen Störungen ein psychosomatischer Zugang über eine Thematisierung der aktuellen psychosozialen Gesamtsituation, Krankheitsverarbeitung, früheren Krankheitserfahrungen und mögliche schwerwiegende Auslösesituationen. In der Praxis steht als anfängliches Ziel ein Ernstnehmen und Verständnis der Beschwerden im Vordergrund. Dabei geht die Gesprächsführung weg vom Symptom in Richtung psychosozialer Aspekte einschließlich Indikation, Motivation sowie kausale Therapieeinleitung der psychosozialen Störung. Vor dem unkritischen Einsatz der Lifestyle-Medizin bei psychischen Störungen muss gewarnt werden, und steht die Therapie der psychosozialen Störung (Psychotherapie, Psychopharmaka) im Vordergrund.

\section{Literatur}

${ }^{1}$ Harth W, Gieler U. Psychosomatische Dermatologie. Heidelberg: Springer, 2005: $183-184$

${ }^{2}$ Gesellschaft für ästhetische Chirurgie Deutschland. Top 10 der Schönheitsoperationen.www.dgac.de

${ }^{3}$ Lehnhardt M, Homann HH, Druecke D, Steinstraesser L, Steinau HU. Liposuktion - Kein Problem ? Chirurg 2003; 74: 808-814

${ }^{4}$ Crisp AH. Dysmorphophobia and the search for cosmetic surgery. $\mathrm{Br}$ Med J (Clin Res Ed) 1981; 282: 1099-1100
${ }^{5}$ Cotterill JA. Body dysmorphic disorder. Dermatol Clin 1996; 14: 457 463

${ }^{6}$ Altamura C, Paluello MM, Mundo E, Medda S, Mannu P. Clinical and subclinical body dysmorphic disorder. Eur Arch Psychiatry Clin Neurosci 2001; 251: 105-108

${ }^{7}$ Sass H, Wittchen H-U, Zaudig M. Diagnostisches und Statistisches Manual Psychischer Störungen DSM-IV. Berlin: Hogrefe, 2001

${ }^{8}$ Stangier U. Hautkrankheiten und körperdysmorphe Störung. Göttingen: Hogrefe, 2002

${ }^{9}$ Bohne A, Keuthen NJ, Wilhelm S, Deckersbach T, Jenike MA. Prevalence of symptoms of body dysmorphic disorder and its correlates: a cross-cultural comparison. Psychosomatics 2002; 43: 486-490

${ }^{10}$ Glaser DA. Kaminer MS. Body dysmorphic disorder and the liposuction patient. Dermatol Surg 2005; 31: 559-561

${ }^{11}$ Veale D, Riley S. Mirror, mirror on the wall, who is the ugliest of them all? The psychopathology of mirror gazing in body dysmorphic disorder. Behav Res Ther 2001; 39: 1381 - 1393

12 Zylka-Menhorn V. Fernsehen: Den TV-,,Schönheitschirurgen“ das Skalpell entziehen. Deutsches Ärzteblatt 2004; 101: 2734-2735

${ }^{13}$ Egle UT. Somatoform disorders an update. MMW Fortschr Med 2005; 17; 147 (Spec 2): 4-6

${ }^{14}$ Mackley CL. Body dysmorphic disorder. Dermatol Surg 2005; 31: 553 558

${ }^{15}$ Phillips KA, Coles ME, Menard W, Yen S, Fay C, Weisberg RB. Suicidal ideation and suicide attempts in body dysmorphic disorder. J Clin Psychiatry 2005; 66: $717-725$

${ }^{16}$ Bürgy M. Dysmorphophobie. Das Sich selbst fremd werden als Störung der Kommunikation. Nervenarzt 1998; 69: 446 - 450

17 Küchenhoff J. Der psychogen motivierte Operationswunsch. Chirurg 1993; 64: $382-386$

18 Menninger KA. Polysurgery and Polysurgical Addiction. Psychoanalyt 1934; 3: 173-199

${ }^{19}$ Siebenmann R, Biedermann K, Maire R, Oelz O, Largiader F. Mania Operativa. Schweiz Rundschau Med 1984; 73: 1215-1221

${ }^{20}$ Hontschik B, Uexküll U. Psychosomatik in der Chirurgie. Stuttgart: Schattauer Verlag, 1999

${ }^{21}$ Harth W, Wendler M, Linse R. Lifestyle-Medikamente und körperdysmorphe Störungen. Ein neues medizinisches Phänomen am Beispiel der Dermatologie. Deutsches Ärzteblatt 2003; 100: 128-131

${ }^{22}$ Hesselink JM. Surfen mit Nebenwirkungen: Probleme rund um die Smartdrugs. Dtsch Med Wochenschr 1999; 124: 707-710

${ }^{23}$ Assmann T, Arens A, Becker-Wegerich P, Schuppe H-C, Lehmann P. Acne fulminans mit sternoklavikulären Knochenläsionen und Azoospermie nach Abusus anaboler Steroide. Z Hautkr 1999; 74: 570-572

${ }^{24}$ Kadison R. Getting an edge - use of stimulants and antidepressants in college. N Engl J Med 2005; 353: 1089-1091

${ }^{25}$ Hengeveld MW. Somatization of erectile disorders by the introduction of sildenafil. Ned Tijdschr Geneeskd 1998; 142: 2333-2336

${ }^{26}$ Pomeranz H, Bhavsar A. Nonarteritic Ischemic Optic Neuropathy Developing Soon After Use of Sildenafil (Viagra): A Report of Seven New Cases. J Neuro-Ophthalmol 2005; 25: 9- 13

${ }^{27}$ Harth W, Linse R. Botulinophilia: contraindication for therapy with botulinum toxin. Int J Clin Pharmacol Ther 2001; 39: 460-463

${ }^{28}$ Brosig B, Kupfer J, Niemeier V, Gieler U. The Dorian Gray Syndrome: psychodynamic need for hair growth restorers and other fountains of youth. Int J Clin Pharm Therap 2001; 39: 279-283 